\title{
Antibiotic Susceptibility of Bacterial Isolates from Pus Specimens Collected from a General Hospital in Dhaka, Bangladesh
}

\author{
Sumona Rahman Shewly ${ }^{1}$, Mosa. Hafsa Akter Ane ${ }^{1}$, Tamanna Zerin ${ }^{1 *}$, Md. Altaf Hossain ${ }^{2}$ \\ ${ }^{1}$ Department of Microbiology, Stamford University Bangladesh, 51, Siddeswari Road, Dhaka-1217, Bangladesh \\ ${ }^{2}$ Insaf Barakah Kidney and General Hospital, 11, Shahid Tajuddin Ahmed Sharani, Dhaka-1217, Bangladesh \\ *Corresponding author
}

\begin{abstract}
Antibiotic resistance and its rapid spread among pathogenic bacterial isolates are regarded as major public health issues around the world. The study aims to detect pyogenic bacteria in pus samples and assess their antibiotic susceptibilities to various antibiotics commonly employed in chemotherapeutic interventions. This is a retrospective study in which 297 pus samples were obtained from patients in the year 2018 to 2020 for aerobic culture and sensitivity testing. In total, $32 \%(95)$ of the samples yielded positive results, whereas, $68 \%$ (202) yielded negative results. The most commonly occurring pathogen was $E$. coli (65) followed by Pseudomonas spp. (14), Staphylococcus aureus (9), Klebsiella spp. (4) and Acinetobacter spp. (3). Patients aged 51 and up were the ones who were largely afflicted by the pus infection. Males were shown to be more susceptible to infection than females. Amikacin, Ceftazidime, Meropenem, Cefotaxime, Netilmicin, Mecillinam, and Fosfomycin were the most susceptible drugs for Klebsiella spp., followed by Meropenem for $E$. coli, Cloxacillin, Doxacillin, Cefotaxime, and Amoxiclave for S. aureus. However, Pefloxacin and Amoxiclav were both effective against Pseudomonas spp., while Levofloxacin, Ciprofloxacin, Doxacillin, Tazobactam+Piperacillin, Imipenem, Netilmicin, and Trimethoprim were effective against Acinetobacter spp. The antibiotic, Ampicillin was found to be resistant to all of the microorganisms tested. In general, most of the isolates were found to be resistant to the majority of the antibiotics. As a result of our research, clinicians will be able to make better decisions when selecting appropriate antibiotics, which aid in patient care and judicious usage, preventing the development of drug resistance in those who are already susceptible.
\end{abstract}

Key words: Pus, Antibiogram, Age, Gender, Bangladesh

\section{INTRODUCTION}

$\mathrm{P}$ us is usually caused by a bacterial infection that is contained in an abscess that can be developed anywhere in the body. Furthermore, virus, protozoa, fungus can also cause wound infection, and sometimes, they simultaneously causes infection with one or more bacteria in a distinct wound [1]. Typically, it looks like white to yellow fluid made up of dead neutrophils, cellular waste, and necrotic tissues. Pus is formed when microbial pathogens cause human skin and soft tissue infections (SSTIs) during or after trauma, burn injuries, and surgical procedures [2-4]. Hospital-acquired wound infections may cause severe morbidity, and prolonged hospitalization that may pose a considerable monetary burden [5]. Both aerobic and anaerobic, gram-positive and gram-negative bacteria are implicated in pus infection. A number of commonly involved bacterial species in pus formation includes Staphylococcus aureus, Staphylococcus epidermidis, Streptococcus pyogenes, Escherichia coli, Streptococcus pneumoniae, Klebsiella pneumoniae, Salmonella typhi, Pseudomonas aeruginosa, Neisseria gonorrhoeae, Actinomyces, Burkholderia mallei, Mycobacterium tuberculosis [6]. However, the rapid development of antibiotic resistance among pathogenic bacteria regarded as a major public health issue around the world. Multidrug-resistant gram-negative bacteria as Acinetobacter baumannii, E. coli, Klebsiella pneumoniae, Pseudomonas aeruginosa, and grampositive methicillin-resistant Staphylococcus aureus (MRSA) progressively associated with nosocomial pus infections over the last few decades due to extensive antibiotic use through self-medication, mis-prescription and inadequate dosage regimens [7-9]. Owing to restricted treatment options and hesitant development of new antibiotic groups, the rapid emergence of multidrug-resistant bacteria poses a significant challenge to global public health. The aims of this research are to characterize pyogenic bacteria found in pus samples, and evaluate their antibiotic susceptibilities to different generations of antibiotics widely used in chemotherapeutic interventions.

\section{MATERIALS AND METHODS}

This is a retrospective study in which 297 pus samples were collected for aerobic culture and sensitivity testing from the patients of Insaf Barakah Kidney and General Hospital, Dhaka from May 2018 to November 2020.

Pus samples were obtained using sterile disposable cotton swabs and aspirates were collected in syringe. Following collection, the specimens were immediately transported and processed in the microbiology laboratory. Blood agar, MacConkey agar and Chocolate agar media were used to inoculate the specimens. The cells were incubated in an aerobic state at $37^{\circ} \mathrm{C}$ for 24 to 48 hours.

After incubation, the bacteria from positive cultures were detected using a standard microbiological technique that 
included hanging drop motility monitoring preparation, gram staining, biochemical reactions such as catalase, oxidase, coagulase, indole, methyl red, voges-proskauer, citrate, urease, and phenyl pyruvic acid tests. All the isolates were tested for antibiotic sensitivity using Kirby Bauer's disc diffusion method on Muller Hinton agar medium and the results were interpreted according to CLSI (Clinical and Laboratory Standards Institute) guidelines. The results were presented in sensitivity [10]. Both the biochemical tests and disc diffusion tests were performed with the standard organisms that were taken as positive controls for each of the individual experiments.

\section{RESULTS}

\section{Distribution of positive and negative samples}

During the study period, 297 samples were processed at the clinical microbiology laboratory in Insaf Barakah Kidney and General Hospital, Dhaka, Bangladesh. The significant bacterial growth confirming the infection is presented at every month in figure $1(\mathrm{~A}, \mathrm{~B}, \mathrm{C})$. The bar diagrams presented the frequency distribution of positive and negative samples in every month of 2018 (A), 2019 (B), and 2020 (C). Our data showed that in almost all of the cases, the number of negative samples was higher than the positive ones.

A.
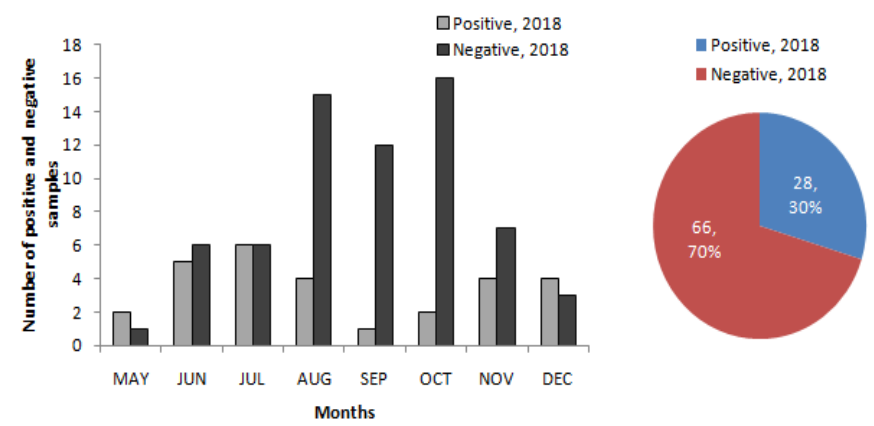

B.

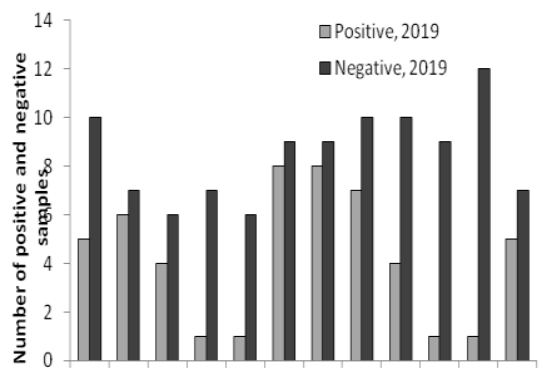

JAN FEB MAR APR MAY JUN JUL AUG SEP OCT NOV DEC Months
C.

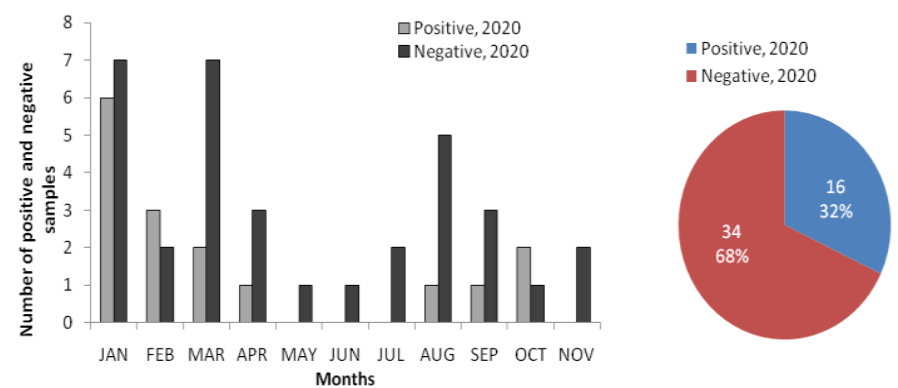

Figure 1: The bar diagram presented the frequency distribution of positive and negative samples in every month of the year 2018 (A), 2019 (B), and 2020 (C). The pie diagram presented the percentage of overall positive and negative samples in the year 2018 (A), 2019 (B), and 2020 (C).

Among the total 297 samples, 32\% (95) showed positive results and 68\% (202) showed no growth (Figure 2).

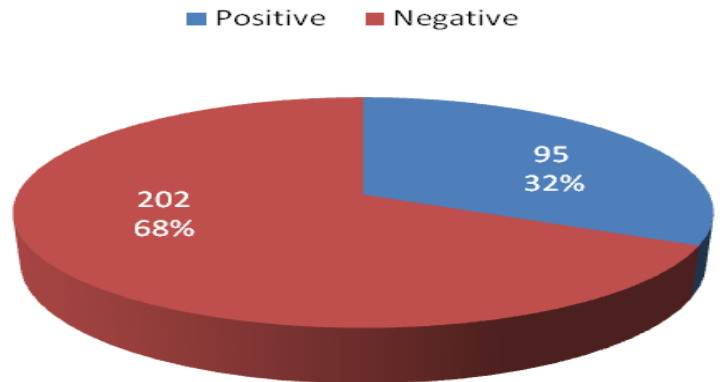

Figure 2: The distribution of the total positive and negative samples obtained from pus samples were presented by pie diagram.

Age- and gender-wise distributions of positive samples

In this study, males were $58.9 \%$ (62 out of 95) and females were $31.35 \%$ (33 out of 95) belonging to 0-80 years of age. This was statistically significant $(\mathrm{P}<0.05)$. Overall, our study showed that males were more prone to pyogenic infection than females. The patients with the ages $21-51^{+}$were highly affected with pyogenic wound infections and $51^{+}$was the most affected age group. Moreover, the number of infected patients was mostly increased with the increasing ages (Figure $3)$.

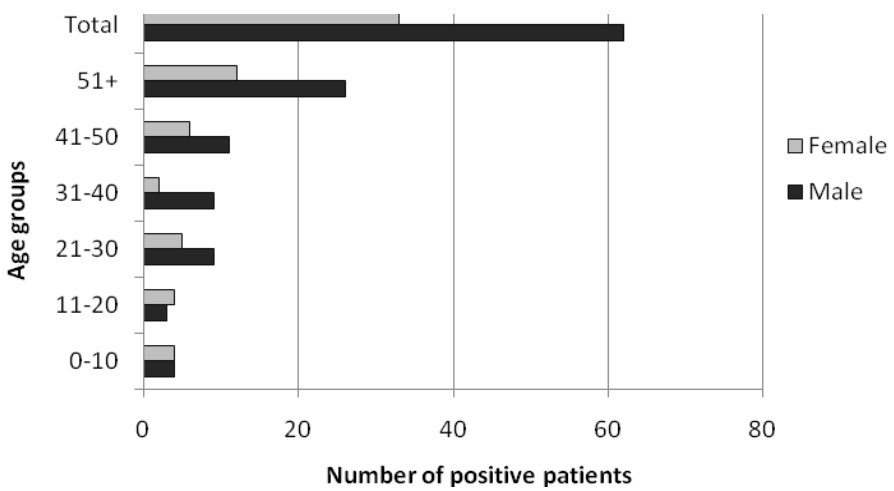

Figure 3: The bar graph showing the age-wise distribution of male and female patients in the study period. 
Frequency distribution of bacterial isolates among infected patients

A total of 95 bacterial pathogens were recovered with the predominance of gram-negative bacteria (86, 90.5\%) where, Escherichia coli $(65,68.42 \%)$ was the leading bacterial pathogen followed by Pseudomonas spp. (14, $14.74 \%)$, Klebsiella spp. $(04,4.21 \%)$ and Acinetobacter spp. $(03,3.16 \%)$. The only gram-positive bacterial species was Staphylococcus spp. $(09,9.47 \%)$ (Table 1). Single culture infection was observed among all the positive patients.

Table 1: Occurrence of bacterial isolates among infected patients

\begin{tabular}{|c|c|c|}
\hline Bacterial isolates & Number & $\%$ \\
\hline E. coli & 65 & 68.42 \\
\hline Pseudomonas spp. & 14 & 14.74 \\
\hline Staphylococcus spp. & 9 & 9.47 \\
\hline Klebsiella spp. & 4 & 4.21 \\
\hline Acinetobacter spp. & 3 & 3.16 \\
\hline
\end{tabular}

\begin{tabular}{|c|c|c|}
\hline Total & 95 & 100 \\
\hline
\end{tabular}

Sensitivity pattern of bacterial isolates

The antibiogram revealed that Amikacin, Ceftazidime, Meropenem, Cefotaxime, Netilmicin, Mecillinam and Fosfomycin were the most susceptible drugs (100\%) for Klebsiella spp. E. coli showed the highest sensitivity $(69.35 \%)$ towards Meropenem, Pseudomonas spp. showed $100 \%$ sensitivity against Pefloxacin, and Amoxiclave; and Acinetobacter spp. showed the same against Levofloxacin, Ciprofloxacin, Doxacillin, Tazobactam+Piperacillin, Impenem, Netilmicin, and Trimethoprim. The only grampositive bacteria Staphylococcus spp. showed the most sensitivity (100\%) towards Cloxacillin, Doxacillin, Cefotaxime, and Amoxiclav. All the bacteria present in the study showed resistance against the antibiotic, Ampicillin. Moreover, most of the bacteria showed a degree of resistance against most of the antibiotics.

Table 2: Antibiotic sensitivity of bacterial isolates against a pool of antibiotics.

\begin{tabular}{|c|c|c|c|c|c|c|c|c|c|c|c|c|c|c|c|c|c|c|c|c|c|c|c|c|c|c|c|c|c|c|c|c|c|c|c|}
\hline Isolates & 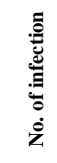 & 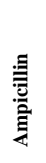 & 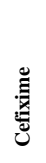 & 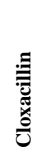 & 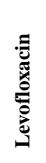 & 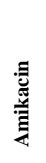 & 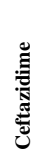 & 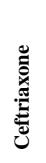 & 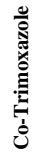 & 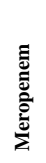 & 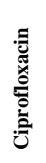 & 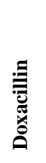 & 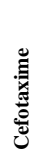 & $\begin{array}{l}0 \\
0 \\
0\end{array}$ & 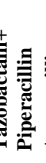 & 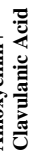 & 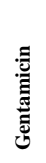 & 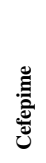 & 音 & 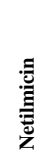 & 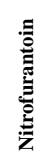 & 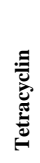 & 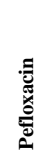 & 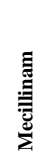 & 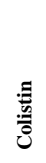 & 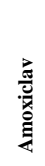 & 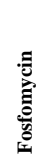 & 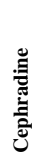 & 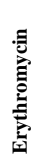 & 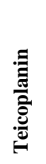 & 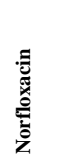 & 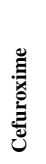 & 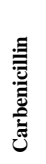 & 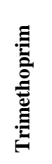 & 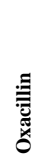 \\
\hline Klebsiella spp. & 4. & $\begin{array}{l}Ð \\
8 \\
\delta\end{array}$ & $\begin{array}{l}\hat{\infty} \\
\stackrel{0}{\circ} \\
\text { ळे }\end{array}$ & $\begin{array}{l}\text { d } \\
8\end{array}$ & 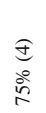 & $\begin{array}{l}\text { 于 } \\
\stackrel{0}{\circ} \\
\varrho\end{array}$ & $\begin{array}{l}\text { 于 } \\
\stackrel{0}{\circ} \\
\stackrel{0}{0}\end{array}$ & $\begin{array}{l}\widehat{0} \\
\circ \\
\hat{6} \\
0 \\
0\end{array}$ & 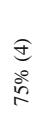 & $\begin{array}{l}\text { 于 } \\
\stackrel{\circ}{\circ} \\
\stackrel{0}{0}\end{array}$ & 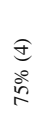 & $\begin{array}{l}\hat{0} \\
8 \\
8\end{array}$ & $\begin{array}{l}\text { d } \\
\circ \\
0 \\
0\end{array}$ & $\begin{array}{l}\text { d } \\
8 \\
8\end{array}$ & $\begin{array}{l}\widehat{0} \\
\circ \\
0 \\
0 \\
0\end{array}$ & $\begin{array}{l}\text { ले } \\
\stackrel{0}{0}\end{array}$ & $\begin{array}{l}\hat{\infty} \\
\stackrel{0}{0} \\
\text { ले } \\
\dot{m}\end{array}$ & $\begin{array}{l}\widehat{d} \\
\stackrel{0}{\circ} \\
\text { in }\end{array}$ & 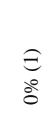 & $\begin{array}{l}\widehat{\Xi} \\
\delta \\
\delta\end{array}$ & $\begin{array}{l}\tilde{E} \\
\stackrel{\Xi}{\Xi} \\
8^{\circ}\end{array}$ & 合 & 令 & 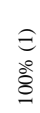 & 之 & 之 & $\begin{array}{l}\widehat{\Xi} \\
\stackrel{0}{\circ} \\
\stackrel{0}{0}\end{array}$ & 令 & 合 & 令 & 立 & $\begin{array}{l}\hat{\Xi} \\
8\end{array}$ & 合 & 会 & 令 \\
\hline E. coli & 65 & $\begin{array}{l}\stackrel{d}{0} \\
\stackrel{0}{0}\end{array}$ & $\begin{array}{l}\widehat{\hat{n}} \\
\stackrel{0}{\circ} \\
\infty \\
\infty \\
0\end{array}$ & $\begin{array}{l}\widehat{0} \\
0 \\
\circ \\
5 \\
0 \\
0\end{array}$ & $\begin{array}{l}\hat{6} \\
0 \\
\circ \\
0 \\
0 \\
0 \\
+\end{array}$ & $\begin{array}{l}\widehat{\hat{n}} \\
\stackrel{0}{0} \\
\dot{0} \\
\dot{0} \\
\ddot{n}\end{array}$ & $\begin{array}{l}\widehat{Q} \\
\stackrel{0}{0} \\
\infty \\
\infty \\
\stackrel{+}{q}\end{array}$ & 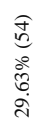 & $\begin{array}{l}\widehat{\vec{\sigma}} \\
0 \\
\frac{0}{2} \\
i n \\
i n\end{array}$ & 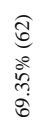 & 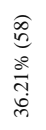 & 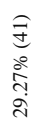 & 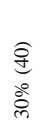 & $\begin{array}{l}\sqrt{2} \\
\stackrel{0}{0} \\
\vdots \\
0 \\
0\end{array}$ & $\begin{array}{l}\sqrt{n} \\
0 \\
0 \\
0 \\
0 \\
0\end{array}$ & 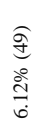 & 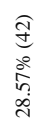 & $\begin{array}{l}\hat{\infty} \\
\stackrel{0}{0} \\
\infty \\
\infty \\
\infty \\
\infty\end{array}$ & $\begin{array}{l}\hat{\theta} \\
\circ \\
\dot{0} \\
\dot{J}\end{array}$ & $\begin{array}{l}\widehat{\varrho} \\
\stackrel{0}{0} \\
\stackrel{0}{0}\end{array}$ & 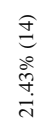 & 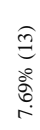 & $\begin{array}{l}\hat{\Xi} \\
\delta^{\circ}\end{array}$ & 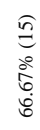 & $\begin{array}{l}6 \\
\therefore \\
\circ\end{array}$ & $\begin{array}{l}5 \\
8 \\
0\end{array}$ & $\begin{array}{l}0 \\
\stackrel{0}{0} \\
0 \\
0 \\
\infty \\
0\end{array}$ & $\begin{array}{l}\hat{\theta} \\
\stackrel{0}{0}\end{array}$ & $\begin{array}{l}\widehat{\hat{a}} \\
\stackrel{0}{0}\end{array}$ & 令 & $\begin{array}{l}\widehat{d} \\
\therefore \\
\circ\end{array}$ & 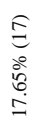 & $\begin{array}{l}a \\
\frac{d}{0} \\
\delta^{\circ}\end{array}$ & 乏 & $\begin{array}{l}\hat{\jmath} \\
8\end{array}$ \\
\hline $\begin{array}{l}\text { Pseudomonas } \\
\text { spp. }\end{array}$ & 14 & $\begin{array}{l}\text { a } \\
8 \\
8\end{array}$ & $\begin{array}{l}\widehat{O} \\
\stackrel{0}{0}\end{array}$ & $\begin{array}{l}5 \\
8 \\
8\end{array}$ & $\begin{array}{l}\widehat{\widehat{d}} \\
\stackrel{0}{0} \\
\stackrel{\sim}{1} \\
\stackrel{0}{0}\end{array}$ & 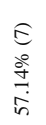 & 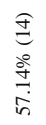 & 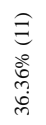 & $\begin{array}{l}\stackrel{\Theta}{0} \\
\stackrel{\circ}{0} \\
\stackrel{0}{0}\end{array}$ & $\begin{array}{l}\widehat{\widehat{l}} \\
\stackrel{0}{0} \\
\frac{0}{6} \\
\stackrel{0}{0}\end{array}$ & $\begin{array}{l}\text { f } \\
\stackrel{0}{0} \\
\text { ì } \\
\text { d }\end{array}$ & $\begin{array}{l}\hat{\infty} \\
\stackrel{0}{0} \\
\vec{d}\end{array}$ & $\begin{array}{l}\hat{\sigma} \\
\stackrel{0}{\infty} \\
m \\
\infty\end{array}$ & $\begin{array}{l}\text { d } \\
8 \\
8\end{array}$ & $\begin{array}{l}\stackrel{\varrho}{\varrho} \\
\stackrel{\circ}{\circ}\end{array}$ & $\begin{array}{l}\sigma \\
\delta \\
\delta\end{array}$ & $\begin{array}{l}\hat{\theta} \\
\stackrel{0}{\circ} \\
\stackrel{+}{q}\end{array}$ & $\begin{array}{l}\widehat{O} \\
\stackrel{0}{0} \\
\text { in }\end{array}$ & $\begin{array}{l}\hat{\sigma} \\
\stackrel{0}{\circ} \\
\stackrel{\sigma}{\sigma}\end{array}$ & $\begin{array}{l}\widehat{0} \\
0 \\
0 \\
0 \\
0\end{array}$ & $\begin{array}{l}0 \\
8 \\
8\end{array}$ & 令 & $\begin{array}{l}\hat{\Xi} \\
\delta \\
\delta \\
\delta\end{array}$ & $\begin{array}{l}\hat{\theta} \\
\text { के } \\
\text { ले } \\
\text { ले }\end{array}$ & $\begin{array}{l}\ominus \\
\triangleq \\
8\end{array}$ & $\begin{array}{l}\hat{\Xi} \\
\stackrel{0}{0} \\
0\end{array}$ & $\begin{array}{l}0 \\
8 \\
0 \\
0\end{array}$ & 令 & 令 & 令 & 令 & $\begin{array}{l}\hat{0} \\
8 \\
8\end{array}$ & 合 & 之 & 之 \\
\hline $\begin{array}{l}\text { Acinetobacter } \\
\text { spp. }\end{array}$ & 3 & $\begin{array}{l}Ð \\
\stackrel{0}{0}\end{array}$ & $\begin{array}{l}\hat{0} \\
\stackrel{0}{0}\end{array}$ & $\begin{array}{l}\text { d } \\
8\end{array}$ & 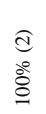 & 之 & $\begin{array}{l}\text { ad } \\
8 \\
8\end{array}$ & 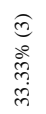 & 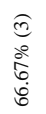 & 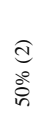 & $\begin{array}{l}\widehat{0} \\
\vdots \\
0 \\
0\end{array}$ & $\begin{array}{l}\widehat{0} \\
\stackrel{0}{0} \\
\stackrel{0}{0}\end{array}$ & $\begin{array}{l}\widehat{0} \\
\stackrel{0}{0}\end{array}$ & $\begin{array}{l}Ð \\
\stackrel{0}{\circ}\end{array}$ & $\begin{array}{l}\widehat{Ð} \\
\stackrel{0}{0} \\
0\end{array}$ & $\begin{array}{l}Ð \\
\stackrel{0}{0}\end{array}$ & $\begin{array}{l}\text { d } \\
8 \\
0 \\
0\end{array}$ & $\begin{array}{l}\widehat{0} \\
\frac{0}{0} \\
5 \\
0 \\
0\end{array}$ & $\begin{array}{l}Ð \\
\delta \\
\delta\end{array}$ & $\begin{array}{l}\widehat{\Xi} \\
\delta \\
\delta \\
\delta\end{array}$ & 合 & 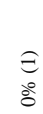 & 令 & 之 & 立 & 立 & 乏 & 合 & 令 & 合 & 合 & 会 & 合 & $\begin{array}{l}\hat{0} \\
\stackrel{0}{\circ} \\
\stackrel{0}{0}\end{array}$ & 合 \\
\hline $\begin{array}{l}\text { Staphylococcus } \\
\text { spp. }\end{array}$ & 9 & $\begin{array}{l}Ð \\
\stackrel{0}{0}\end{array}$ & 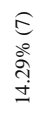 & $\begin{array}{l}0 \\
0 \\
\circ \\
0\end{array}$ & $\begin{array}{l}E \\
E \\
\stackrel{0}{*} \\
\infty \\
\infty\end{array}$ & $\begin{array}{l}\hat{n} \\
0 \\
0 \\
0\end{array}$ & $\begin{array}{l}\text { 于 } \\
8 \\
8\end{array}$ & $\begin{array}{l}0 \\
0 \\
0 \\
0 \\
0\end{array}$ & $\begin{array}{l}\hat{a} \\
\stackrel{0}{\circ} \\
\stackrel{0}{\circ} \\
\stackrel{-}{\therefore}\end{array}$ & $\begin{array}{l}\widehat{0} \\
\circ \\
\hat{6} \\
0 \\
0\end{array}$ & $\begin{array}{l}\hat{\infty} \\
\stackrel{0}{0} \\
\stackrel{0}{n} \\
m\end{array}$ & $\begin{array}{l}E \\
\stackrel{0}{\circ} \\
\delta\end{array}$ & $\begin{array}{l}\widehat{0} \\
\stackrel{0}{0} \\
0\end{array}$ & 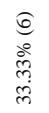 & $\begin{array}{l}E \\
0 \\
0 \\
\infty \\
i \\
\text { i }\end{array}$ & $\begin{array}{l}E \\
\text { E } \\
\text { d } \\
\text { in } \\
\text { in }\end{array}$ & 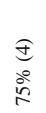 & $\begin{array}{l}6 \\
\infty \\
\infty \\
m \\
m \\
m\end{array}$ & 令 & 合 & $z$ & $\begin{array}{l}\text { वी } \\
0 \\
0 \\
0 \\
0\end{array}$ & 之 & $\bar{z}$ & 乏 & $\begin{array}{l}\widehat{Ð} \\
8 \\
\delta \\
\delta\end{array}$ & 立 & $\begin{array}{l}\hat{\Xi} \\
\stackrel{0}{0}\end{array}$ & $\begin{array}{l}\hat{\Xi} \\
8_{0}^{\circ}\end{array}$ & $\begin{array}{l}\hat{\Xi} \\
\therefore\end{array}$ & $\begin{array}{l}\widehat{d} \\
\therefore \\
\therefore\end{array}$ & 会 & 合 & 之 & 乏 \\
\hline
\end{tabular}

\section{DISCUSSION}

The present study revealed $E$. coli to be the most commonly occurring pathogen in pus samples which is in agreement with a previous study [11]. Another study conducted in Nepal,
Kathmandu also reported $E$. coli to be the most frequently isolated bacteria in pus infection [12]. However, S. aureus was the most common pathogen studied in earlier studies [13, 14]. Among 95 bacterial isolates, 69 were from the Enterobacteriaceae family which includes E. coli (65) and 
Klebsiella spp. (04). Pseudomonas spp. was found to be the second commonly occurring pathogen in wound infections. One study by Basu et al. reported that Pseudomonas spp. and E. coli to be the most common pathogens in pus infection which is consistent with our study [15]. However, in this study, positive samples were lower in number than negative samples which were contrary to the results of Trojan, Razdan et al. 2016 [11].

The month-wise distribution of samples showed that the maximum infection was observed in the months June, July, and August in 2018 and 2019. High humidity in the air during monsoon season from June to October might be the reason for the high infection rate by bacteria or viruses. But, in 2020, the number of positive samples were gradually decreased in accordance with the total samples from Jan to Apr. With a very low number of samples, no growth was observed in the specimens of May, Jun, Jul, and Nov. Besides, total samples were also very low in 2020 in comparison to the other two years. The reason behind is the COVID 19 pandemic situation as the government imposed a total lockdown from March 26, 2020 and it continued for long time and also, at the beginning, people were very afraid of going outside unless its an awfully urgent situation $[16,17]$.

Among 95 infected patients, $62(58.9 \%)$ were males and 33 $(31.35 \%)$ were females. The higher number of male patients than female patients correlates our result with earlier studies [18-23]. Age is a significant risk factor in the occurrence of any infection. Our results showed patients with the age of $51^{+}$ were mostly infected with pathogens and got pus formation which coincides with several previous findings [24, 25].

Most of the bacteria were found to have a high degree of resistance towards $3^{\text {rd }}$ and $4^{\text {th }}$ generation penicillin and cephalosporin. The antibiotics that are used for repeated empirical treatment might be the reason for the development of high antibiotic resistance. Klebsiella spp. showed 50\%, E. coli showed 37.93\%, Pseudomonas spp. showed 50\%, Acinetobacter spp. showed $66.67 \%$ and Staphylococcus spp. showed $33.33 \%$ sensitivity towards $4^{\text {th }}$ generation Cefepime. Whereas, a better sensitivity was observed towards Tazobactam/Piperacillin which is an alternative to $3^{\text {rd }}$ generation Cephalosporins and Carbapenems that showed $66.67 \%$ sensitivity by Klebsiella spp., $61.82 \%$ by E. coli, $90 \%$ by Pseudomonas spp., $100 \%$ by Acinetobacter spp. and $42.86 \%$ by Staphylococcus spp. A number of antibiotics were found effective against specific microorganisms. Overall, Carbapenems (Meropenem) are still sensitive to growing resistance in both gram-positive and gram-negative bacteria tested which is nearly related to earlier studies [13, 26]. However, all the bacteria present in the study showed complete resistance against the $2^{\text {nd }}$ generation penicillin, Ampicillin.

\section{CONCLUSION}

The pyogenic infection has long been a leading cause of morbidity and mortality. Emerging multidrug-resistant strains are a significant source of concern in the treatment of these diseases. Appropriate and judicious antibiotic selection based on antibiotic sensitivity data will restrict the emergence of drug-resistant strains in the future, allowing for effective treatment of these clinical conditions. As a result of our research, the clinician will be able to make better decisions in choosing suitable antibiotics that will not only help in treatment but will also help with judicious use in avoiding the development of drug resistance in those that are already susceptible.

\section{REFERENCES}

[1] D. Hailu, D. Mekonnen, A. Derbie, W. Mulu and B. Abera, "Pathogenic bacteria profile and antimicrobial susceptibility patterns of ear infection at Bahir Dar Regional Health Research Laboratory Center, Ethiopia," Springerplus vol. 16, no. 5, pp. 466, 2016.

[2] A. L. Cogen, V. Nizet and R. L. Gallo, "Skin microbiota: a source of disease or defence?" British Journal of Dermatology, vol. 158, no. 3, pp. 442-455, 2008.

[3] M. S. Dryden, "Complicated skin and soft tissue infection," Journal of Antimicrobial Chemotherapy, vol. 65, supplement 3, pp. iii35-iii44, 2010.

[4] A. Scalise, A. Bianchi, C. Tartaglione et al., "Microenvironment and microbiology of skin wounds: the role of bacterial biofilms and related factors," Seminars in Vascular Surgery, vol. 28, no. 34, pp. 151-159, 2015.

[5] P. G. Bowler, B. I. Duerden and D. G. Armstrong, "Wound microbiology and associated approaches to wound manage $\square$ ment," Clinical Microbiology Reviews, vol. 14 no. 2, pp. 244-269, 2001.

[6] A. Thompson, A. Miles, Alexander, (1921). "Pyogenic Bacteria". Manual of Surgery (6th ed.). Oxford Medical Publications. OCLC 335390813.

[7] L. B. Rice, "Antimicrobial resistance in gram-positive bacteria," The American Journal of Medicine, vol. 119, no. 6, supplement 1, pp. S11-S19, 2006.

[8] A. M. Misic, S. E. Gardner and E. A. Grice, "The Wound Microbiome: modern approaches to examining the role of microorganisms in impaired chronic wound healing," Advances in Wound Care, vol. 3, no. 7, pp. 502-510, 2014.

[9] J. Iredell, J. Brown and K. Tagg, "Antibiotic resistance in Enter $\square$ obacteriaceae: mechanisms and clinical implications," British Medical Journal, vol. 352, Article ID. h6420, 2016.

[10] S. Duggal, P. Khatri, R. Parihar and R. Arora, "Antibiogram of various bacterial isolates from pus samples in a tertiary care centre in Rajasthan," International Journal of Science and Research, vol. 4, no. 5, pp. 1580-1584, 2015.

[11] R. Trojan, L. Razdan and N. Singh, "Antibiotic Susceptibility Patterns of Bacterial Isolates from Pus Samples in a Tertiary Care Hospital of Punjab, India," International Journal of Microbiology, vol. 2016, Article ID 9302692, 2016.

[12] M.S. Raza, A. Chander and A. Ranabhat, "Antimicrobial susceptibility patterns of bacterial isolates in postoperative wound infections in a tertiary care hospital, Kathmandu, Nepal," Open Journal of Medical Microbiology, vol. 3, no. 3, pp.159-63, 2013.

[13] D. R. Rao, R. Basu and D. R. Biswas, "Aerobic Bacterial Profile and Antimicrobial Susceptibility Pattern of Pus Isolates in a South Indian Tertiary Care Hospital," IOSR Journal of Dental and Medical Sciences, vol. 13, no. 3, pp. 59-62, 2014.

[14] S. Mukherjee, S. Mishra and S. Tiwary, "Microbial profile and antibiogram of pus isolate in a tertiary care hospital of Western Odisha," Journal of Evolution of Medical and Dental Sciences, vol. 9, no. 16, pp. 1325-1330, 2020.

[15] S. Basu, T. Ramchuran Panray, T. Bali Singh, A. K. Gulati and V. K. Shukla, "A prospective, descriptive study to identify the microbiological profle of chronic wounds in outpatients," Ostomy Wound Manage, vol. 55, no. 1, pp. 14-20, 2009. 
[16] "Bangladesh in virtual lockdown as coronavirus fight flares". Bdnews24.com. Archived from the original on 25 March 2020.

[17] "Dhaka wears deserted look as 10-day holiday begins". New Age. Archived from the original on 27 March 2020.

[18] R.A. Khan, M. Jawaid and M. Khaleel. "Bacteriological profile and antibiogram of isolates from pus samples in a tertiary care centre." International Journal of Current Microbiology and Applied Sciences, vol. 7, no. 1, pp. 387-394, 2018.

[19] M. Mama, A. Abdissa and T. Sewunet, "Antimicrobial susceptibility pattern of bacterial isolates from wound infection and their sensitivity to alternative topical agents at Jimma University Specialized Hospital, South-West Ethiopia," Annals of Clinical Microbiology and Antimicrobials, vol. 13, 14, 2014.

[20] M. Taye, "Wound infection in Tikur Anbessa hospital, surgical department," Ethiopian Medical Journal, vol. 43, no. 3, pp. 167174, 2005.

[21] A. Gelaw, S. G. Selassie, M. Tiruneh and M Fentie, "Antimicrobial susceptibility patterns of bacterial isolates from patients withpostoperative surgical site infection, health professionals and environmental samples at a tertiary level hospital, North West Ethiopia," International Journal of Pharmacy \& Industrial Research, vol. 3. No. 1, pp. 1-9, 2013.
[22] N. Goswami, H. R. Trivedi, A. P. Goswami, T. K. Patel and C. B. Tripathi, "Antibiotic sensitivity profile of bacterial pathogens in postoperative wound infections at a tertiary care hospital in Gujarat, India," Journal of Pharmacology \& Pharmacotherapeutics, vol. 2, no. 3, pp. 158- 164, 2011.

[23] O. E. Amoran, A. O. Sogebi and O. M. Fatigues, "Rates and risk fac tors associated with surgical site infections in a tertiary care center in South-Western Nigeria," International Journal of Tropical Disease \& Health, vol 3. No. 1, pp. 25-36, 2013.

[24] A. S. Mundhada and S. Tenpe, "A study of organisms causing surgical site infections and their antimicrobial susceptibility in a tertiary care government hospital," Indian Journal of Pathology and Microbiology, vol. 58, no. 2, pp. 195-200, 2015.

[25] U. O. Ikeanyi, C. N. Chukwuka and T. O. Chukwuanukwu, "Risk fac tors for SSIs following clean orthopaedic operation," Nigerian Journal of Clinical Practice, vol. 16, no. 4, pp. 443-447, 2013.

[26] R. A. Khanam, M. R. Islam, A. Sharif, R. Parveen, I. Sharmin and M. A. Yusuf, "Bacteriological Profiles of Pus with Antimicrobial Sensitivity Pattern at a Teaching Hospital in Dhaka City," Bangladesh Journal of Infectious Diseases, vol. 5, no. 1, pp. $10-14,2018$ 